\title{
THE
}

\section{Understanding the Relationship between Food Variety, Food Intake, and Energy Balance}

Hollie A. Raynor

Maya Vadiveloo

University of Rhode Island, maya_vadiveloo@uri.edu

Follow this and additional works at: https://digitalcommons.uri.edu/nfs_facpubs

The University of Rhode Island Faculty have made this article openly available.

Please let us know how Open Access to this research benefits you.

This is a pre-publication author manuscript of the final, published article.

Terms of Use

This article is made available under the terms and conditions applicable towards Open Access

Policy Articles, as set forth in our Terms of Use.

Citation/Publisher Attribution

Raynor, H.A. \& Vadiveloo, M. Curr Obes Rep (2018) 7: 68. https://doi.org/10.1007/s13679-018-0298-7

Available at: https://doi.org/10.1007/s13679-018-0298-7

This Article is brought to you for free and open access by the Nutrition and Food Sciences at DigitalCommons@URI. It has been accepted for inclusion in Nutrition and Food Sciences Faculty Publications by an authorized administrator of DigitalCommons@URI. For more information, please contact digitalcommonsgroup@uri.edu. 
Understanding the Relationship between Food Variety, Food Intake, and Energy Balance

*Hollie A. Raynor, PhD, RD, LDN ${ }^{\mathrm{a}} \&$ *Maya Vadiveloo, $\mathrm{PhD}, \mathrm{RD}^{\mathrm{b}}$

a Department of Nutrition, University of Tennessee, Knoxville, TN

${ }^{b}$ Department of Nutrition and Food Sciences, University of Rhode Island, Kingston, RI;

maya_vadiveloo@uri.edu; p: 401-874-2002; f: 401-874-5974

*Co-First Author

Corresponding Author: Hollie A. Raynor, Department of Nutrition, 1215 Cumberland Avenue,

229 JHB, Knoxville, TN 37996; hraynor@utk.edu; p: 865-974-6259; f: 865-974-3491

Key Words: Food, Diet, Variety, Energy, Weight, Habituation

Word Count: 4,151

Number of Figures: $0 \quad$ Number of Tables: 1 


\begin{abstract}
Purpose of review: In accordance with US dietary guidance, incorporating variety into the diet can align with energy balance, though greater food variety in some categories may make energy balance more challenging. Thus, experimental and epidemiologic evidence is summarized on the relationship between food variety, food and energy intake, and energy balance.

Recent findings: Lab-based, experimental research consistently demonstrates that greater variety within foods or sensory characteristics of food increases food and energy intake within an eating occasion. Epidemiologic evidence is less consistent, potentially driven by differing methodologies, particularly in defining and measuring food variety. Moreover, the effect of variety on energy balance appears to be moderated by food energy density.

Summary: Integrating insights from experimental and epidemiologic research are essential for strengthening food variety guidance including: developing evidence-based definitions of food variety, understanding moderators of the relationship, and developing practical guidance interpretable to consumers.
\end{abstract}

\title{
Word Count: 145
}




\section{Introduction}

Variety is widely regarded as a determinant of food and energy intake [1] and often, improves dietary quality, which is why variety has been an important component of US dietary guidance [2]. Within- and across-meals, humans habituate to food cues, and evidence consistently suggests that variety promotes food and energy intake by slowing the rate of habituation, or the rate at which food palatability declines [3]. This decrease in habituation rate produces eating occasions in which a greater amount of food is consumed [3]. Thus, if variety enhances intake within- and across-meals, greater variety in the diet may make achieving a healthy state of energy balance more challenging [1]. Considerable research has explored the influence of food variety on gram and energy intake, and more recently, energy balance [4-39]. However, lack of clarity in the definition and measurement of "variety," has limited ability to develop clear recommendations regarding dietary variety.

\section{Historical context and theoretical basis for defining variety}

The historical purpose of recommendations surrounding dietary variety was to ensure adequate intake of all macro- and micronutrients to prevent the development of deficiency diseases and to reduce the likelihood of toxicity associated with excess consumption of certain food groups or tainted food $[2,40]$. Thus, historically, dietary variety was defined primarily with respect to intake of different food groups (i.e. fruits versus grains) or different foods within a group (apples versus oranges), which by definition also varied considerably with respect to macro- and micronutrient content. However, as the food system evolved and deficiency diseases became less concerning in developed countries, the influence of variety on food and energy intake and energy balance drew increased attention with accumulating evidence that the presence 
of food variety encouraged overconsumption [41]. Additionally, this evidence suggested that variety, not only at the nutrient-level (i.e., foods made up of differing levels of macro- or micronutrients) but at the sensory-level (i.e., foods of similar macro- and/or micronutrient content, but made up of different flavors, colors, textures, etc.) influenced intake $[4,8,29,30]$. As a result of this enhanced knowledge, assessing dietary variety has become more complex as it is now important to clarify the point at which variety influences food intake [42] - both within a single eating occasion, as well as during overall usual food intake.

Moreover, the interactions between dietary variety and other factors that influence energy intake require further exploration when evaluating relationships between variety and energy balance. The energy density of foods also has a robust influence on overall energy intake, with foods higher in energy density producing greater energy intake than foods lower in energy density [43]; this suggests that the influence of variety on food consumption, energy intake, and energy balance may differ as a function of the energy density of the foods consumed. For example, greater variety of foods higher in energy density (high-energy-dense [HED] foods) may increase intake of these foods, contributing to greater overall energy intake. Alternatively, greater variety of foods lower in energy density (low-energy-dense [LED] foods) may increase intake of these foods, and due to their energy density may not significantly increase energy intake, or may actually assist with reducing energy intake if increased consumption of LED foods reduces intake of HED foods due to the occurrence of substitution or displacement [44].

\section{The influence of variety on intake- what does the literature currently say?}

Previous research has examined the effect of food variety on intake in several ways, including food variety within one eating occasion, food groups, and the overall diet. The outcomes investigated have included gram and/or energy intake of the meal, food group, or 
overall diet. Within these investigations, a few studies have focused on if food type, such as LED and HED, is an important moderator in the relationship between variety and food and energy intake.

Variety within one eating occasion

The vast majority of research, conducted in children and adults of varying weight and dietary restraint status, investigating the influence of variety within one eating occasion on intake has examined this relationship experimentally in controlled laboratory settings $[4-6,8,10-13,17$, $18,20,23,27-31,33,37,38]$. This body of research has implemented variety typically using one of two paradigms. The first introduces variety within an eating occasion by presenting a new food (as opposed to the same food) across several courses. The second introduces variety within a single course by providing several different foods at once in comparison to presenting a single food (non-variety). These investigations have quantified intake objectively, using weighted intake. In general, when variety occurs via consecutive courses [12, 13, 28-31, 38], greater intake occurs in the variety as compared to the non-variety condition [13, 28-31, 38]. Similarly, greater intake occurs in the variety vs. non-variety condition when food is presented in one course in most $[4-6,8,17,18,20,23,27,33,37]$, but not all $[10,11]$ studies.

While not specifically designed to examine if the type of food influences the effect of variety on intake, a few of these studies have examined the effect of variety on intake using only HED foods, such as ice cream or chocolate candy [4, 8, 10, 38], or LED foods [17, 23, 27], such as fruits and vegetables. The majority of these investigations find that greater variety in HED or LED foods does increase intake of these foods $[4,8,17,23,27,38]$. Greater variety in HED foods has translated to increased energy intake $[4,8,38]$, but greater variety in LED foods has produced mixed outcomes on energy intake [17, 23]. 
These studies have also found that the effect of variety does not just occur when very different foods are provided (i.e., banana, cracker, chocolate), but when provided foods are of similar nutrient composition, but differ in terms of at least two sensory characteristics, such as flavor and color. For example, Rolls and colleagues provided participants with a variety of yogurts differed only in flavoring, coloring, and texture (hazelnut, blackcurrant, and orange), and compared intake in this condition to intake when only one type of yogurt was provided [29]. Results found that when provided with yogurts that varied in flavor, color, and texture, participants consumed more as compared to being provided with just one type of yogurt [29]. Similar findings have occurred with variety achieved through different colors and flavors of ice cream $[4,8]$. However, other studies have not found an effect of variety on intake when variety is achieved by differences in only one sensory characteristic (e.g. differences in color only) [10, 30]. The difference in findings may be that certain sensory characteristics (e.g. food flavor) have a greater influence on food intake than others (e.g. food color or texture) or that more than one sensory characteristic of the food needs to be different to produce enough variety to enhance intake. Importantly, this research suggests that small sensory differences in food may contribute to how variety influences intake.

One study, with adults of varying weight status, has examined the association between variety and energy intake within the evening meal in free-living situations, using self-reported methods [14]. This investigation also found that a greater variety of foods available at dinner was related to greater energy intake at dinner, even if those foods were not consumed, regardless of weight status.

Variety occurring in an eating occasion across several days has also been examined in controlled laboratory settings, in which weighed food intake was obtained $[9,25]$. In these 
investigations, variety is achieved by presenting a different food on each day during the eating occasion (i.e., for a mid-afternoon snack, presenting chips on day 1, cookies on day 2, etc.). The comparison is presenting the same food on each day during the eating occasion (i.e., for a midafternoon snack, presenting chips on day 1,2, etc.). Findings from these studies are mixed, with one study finding no effect of HED food variety on snack intake across days in adults [25], and the other finding greater food and energy intake when differing HED dinner entrées were provided across days to children [9].

One study has examined the effect of variety occurring in an eating occasion across days within free-living situations [9]. This investigation examined variety in HED dinner entrées, defined as different entrées (i.e., macaroni and cheese, fish sticks, chicken nuggets) eaten at home across four weeks, in comparison to no variety in HED dinner entrées (all entrees were macaroni and cheese) and similar HED dinner entrees (all entrees were macaroni and cheese, but had different pasta shapes and sauce flavors) on dinner intake in overweight children [9]. Selfreported dinner intake over the 4 weeks showed that dinner energy intake was lower in the conditions that had no variety or had similar HED entrées [9].

In summary, a large amount of research investigating the effect of variety within one eating occasion has been conducted among adults and children. This research has been predominantly experimental and conducted in laboratory settings, with objective measures of intake obtained. A very limited amount of research regarding the effect of variety within one eating occasion has been conducted in free-living situations. As a whole, this research strongly indicates that having a greater amount of variety within an eating occasion increases intake. This research also suggests that the sensory attributes of food contribute to variety.

Variety within the overall diet 
Research examining food variety and intake within the overall diet has been predominantly observational, with consumption occurring in free-living situations and both variety and intake being measured using self-reported methods $[7,15,16,19,21,26,32,34-36$, 39]. Variety has been quantified as occurring within certain traditional food groups (i.e., fruits and vegetables) or similar types of food combined into a food group (i.e., HED or high-fat foods), or as overall variety within the diet. Regardless of how variety is quantified, the most common energy variable reported is overall energy intake.

When examined cross-sectionally, investigations with children and adults of varying weight status examining variety within certain food groups or types of foods consumed and intake have produced mixed outcomes $[15,16,19,26,39]$. The associations between variety within vegetables (LED foods) in relation to overall energy intake are inconsistent $[15,19]$, while studies examining variety within foods higher in energy density $[15,26]$ or more global definitions of dietary variety $[7,32,34,35]$ have consistently found a positive relationship between variety and overall energy intake. Similarly, research examining the influence of variety on intake and weight status (reflecting energy balance) found that variety in more energy-dense food groups was related to greater energy intake and higher weight status $[15,26]$, while one found that greater "healthful variety" was related to higher energy intake, but lower excess weight and adiposity [35]. Outcomes related to energy balance were more heterogeneous in research examining the association between more global measures of dietary variety, energy intake, and weight status [7, 32, 34].

Longitudinal research on the influence of variety on energy intake is limited, and has only been examined within the context of adult weight loss [21,36]. One investigation examined food group variety, and found that reductions in variety in food groups higher in fat 
were related to decreases in energy intake and weight loss at 18 months [21], while the other found that increases in "healthful variety" were related to greater energy intake, but greater weight loss at 24 months [36].

Similarly, there are few experimental investigations that have tested a food group variety prescription within the context of a lifestyle intervention in adults [22, 24]. Both investigations limited variety in HED food groups (i.e., non-nutrient-dense, high-energy-dense foods such as cookies and chips) and examined if limiting variety in the food group reduced energy intake in these foods, assisting with reducing overall energy intake, thereby enhancing weight loss. Both studies demonstrated that the limited variety prescription could be implemented, and the appropriately powered study found that the limited variety prescription did decrease energy intake from the food group in comparison to the comparison group that did not have a variety prescription [24]. However, the prescription did not appear to enhance overall reductions in total energy intake or weight loss in either study, potentially related to suboptimal compliance with the dietary prescription and/or compensation in other areas of the diet. More research is needed to determine whether concomitant increases in variety within LED foods alongside decreases in variety within HED foods improves adherence to dietary prescriptions [41] .

As a whole, the research in the area of variety within the diet on intake is much less consistent than research regarding variety within one eating occasion. This most likely is due to differing methods of assessing and quantifying dietary variety and energy intake, and perhaps differences in habituation in response to variety over longer periods of time. However, even with the differences, it does appear that variety within HED foods is most consistently related to energy intake and anthropometrics.

\section{Potential mechanism of variety on intake: habituation}


The effect of variety appears to be related to predominantly sensory, and potentially to nutrient, characteristics of food, supporting the hypothesis that the variety effect is due to a basic form of learning, habituation [1, 3, 9, 42, 45-48]. Habituation occurs when with the repeated presentation of the same stimulus (food), behavioral and physiological responses (consumption) decrease $[1,3,48]$. Habituation demonstrates stimulus specificity, meaning that a more rapid rate of habituation occurs when the stimuli that are presented are more similar (i.e., foods of similar flavors, colors, and shapes) [9, 42], and slows when varied stimuli are presented (i.e., foods with differing flavors, colors, and shapes) $[9,46,49,50]$. Thus, an eating occasion with only one food (the non-variety conditions in the previous research), or very similar sensory foods (chocolates of differing colors, but with similar flavors and texture) would produce a more rapid rate of habituation, causing eating to end more rapidly leading to a lower intake, while eating occasions with more varied foods would slow the rate of habituation, leading to a longer bout of consumption and greater intake.

The influence of variety on habituation rate within an eating occasion has been examined in both children and adults, and has consistently demonstrated that variety slows the rate of habituation and increases consumption when measured within one laboratory session (equivalent of an eating occasion) [3]. While limited, evidence is available regarding long-term habituation [9]. One study examined the influence of variety in HED entrées at dinner over 5 days on habituation and dinner energy intake in children with overweight and obesity within a laboratory setting [9]. When children were presented with differing versus the same or a sensory similar HED entrée over the 5 days, the children with greater variety in HED entrees habituated more slowly and consumed more on day 5 at dinner, as compared to the children consuming the same or sensory similar entrées over the 5 days. While these studies were conducted among children 
with overweight, previous research suggests that the rate of habituation to food stimuli varies in lean children as well [51], and factors like HED entrée variety, which reduce habituation, increase the risk of excess weight gain in all children.

\section{Enhancing our understanding of the influence of variety on intake: methodologic considerations}

$\underline{\text { Developing a consistent definition }}$

A consistent definition that quantifies measurement of dietary variety is integral for understanding the relationships between food variety, food intake, and energy balance. Given the different types of study designs and methods of manipulating and assessing variety that have been used in research in this area, several components of variety need to be defined to develop a consistent definition. The first component that requires more clarity is the period over which variety is measured - variety has been measured within a single meal, over a few days, or over periods up to 1-year. It also remains unclear whether variety definitions must specify a minimum frequency of intake when counting the number of unique foods. Also, it is unknown if variety definitions must specify a minimum serving size consumed. Definitions of variety that include shorter time periods, greater frequency of intake, and larger minimum serving sizes (e.g. assessed over one week, with foods needing to be consumed daily, and at least $1 / 2$ serving of a food must be consumed to count toward variety scores) will produce a lower amount of variety in the diet, while definitions that include longer time periods, lower frequency of intake, and smaller (or no) minimum serving sizes consumed will produce a greater amount of variety in the diet (e. g. assessed over 6 months, with foods needing to be consumed weekly, with no minimum serving size required to be consumed to count towards variety scores). Little research has been conducted to ascertain how these components may moderate the effect of variety on intake, but if 
the effect of variety is caused by habituation, one investigation has reported that serving size consumed does not influence habituation rate among children [52], although both portions used in this study would constitute at least 1 serving.

While, period, frequency, and serving size consumed may be important components in defining variety, it has never been clarified within this body of research as to what constitutes variety. Within the existing literature, definitions vary considerably and variety can refer to the number or proportion of (1) foods from different food groups, (2) foods with different macronutrient composition or energy density, (3) different foods within a food group or food item, or (4) the same food with varying sensory qualities. These definitions can then be further classified as the types of variety occurring within an eating occasion, across eating occasions, within food groups, and/or the overall diet.

Defining what constitutes food variety

Key differences between dietary variety definitions center on whether (1) foods are examined at the food or ingredient level, and (2) what sensory, food, or dietary characteristics are used to differentiate a unique food (e.g. macronutrient/energy composition, food groups specified in the U.S. Food Guide Pyramid [i.e. Pyramid Food Groups], food code, or flavor). Variety definitions that rely on a food-based approach assume that foods are perceived as discrete units rather than as multi-ingredient items. As a result, mixed dishes (e.g. chicken soup) are treated as a single food [7, 39]. Variety definitions that rely on an ingredient-based approach assume that variety is more granular, and that mixed dishes like chicken soup are more varied if they have multiple vegetables vs. a single vegetable. As a result, these studies disaggregate mixed dishes [53] into their component ingredients, and count each ingredient toward the variety score. This type of method of assists with quantifying sensory aspects of variety. However, based on 
experimental evidence collected within one eating occasion, variety may be perceived as a combination of a flavor and food-based approach. In this method, mixed dishes may not be broken down into their ingredients, but there is recognition that different flavors of a mixed dish (i.e., pepperoni versus sausage pizza) may contribute to variety, or that the addition of condiments or seasonings to the same mixed dish (plain hamburger versus cheese hamburger) may contribute to variety.

Whether a food-based or ingredient-based approach is used, the characteristics used to differentiate unique foods also needs to be defined. The majority of studies have used food group (e.g. Pyramid Food Groups, food frequency questionnaire (FFQ) defined groups, or food groups correlated with adiposity indices) or food-based definitions, but some studies have defined a unique food based on substantive differences in macronutrient composition or energy density. While the food characteristic(s) used to differentiate between "unique foods" should be informed by the experimental literature, it is important that definitions can easily be measured in free-living settings using available diet assessment methods.

Based on current process-level evidence and available diet assessment tools, the authors recommend using an ingredient-based definition of variety, measuring average daily or within-meal variety over 1-week, specifying no minimum frequency of intake, but requiring that at least $1 / 2$ serving is consumed. Using this definition, researchers can further classify variety within HED and LED foods. It is critical to control for total energy intake within the measurement period among free-living populations given the associations between variety and energy intake and the lack of consideration for larger serving sizes in the proposed definition, which also influence intake. Finally, while the proposed definition is based on emerging evidence from existing research, no one has tested the association between this definition of 
variety and food and energy intake, and energy balance, which is required before any definition is adopted.

\section{Variety and dietary assessment methodology}

Lastly, when assessing variety, the dietary assessment method used will influence results. Food frequency questions (FFQs) have the benefit of assessing longer periods of time (typically 1-mo to 1-year), which is important when trying to evaluate the effect of usual dietary variety on food and energy intake. By definition, FFQs will constrain variety measures because they only incorporate a limited number of foods. Additionally, analyzing dietary data from FFQs requires a more global food-based approach to counting foods since many similar foods are often grouped as a single item, making it challenging to disaggregate foods into their component ingredients. On the other hand, 24-hour dietary recalls and food records are unconstrained with respect to food lists, allowing for the use of food- or ingredient-based variety definitions, but are often constrained with respect to period. Multiple days of observation are necessary to capture usual variation in food intake with 1-day of recall strongly underestimating total variety and 10-days nearly exhausting many people's food repertoire [39]. Because most studies reliant on these methods utilize 1-2 days of recall, they are not typically capturing total variety within the diet, leading to a different source of measurement error in the assessment of dietary variety. Thus, the main methods of dietary assessment have different measurement aspects that affect quantification of variety.

In summary, more research is needed to better understand how best to assess food variety. Greater clarity is needed regarding the period needed for assessment, and the frequency and amount of food consumed that contributes to variety. Furthermore, greater understanding regarding if whether a food-based (e.g. chicken soup) ingredient-based (e.g. chicken, noodles, 
celery, carrots), sensorial-based (e.g. red vs. yellow tomatoes), or some combination of these factors, when defining variety differentially affects the relationship between variety, energy intake, and energy balance is needed. Lastly, the art of communicating evidence-based dietary variety recommendations into practical dietary advice will need to be balanced with the science.

\section{Developing guidance on variety for achieving energy balance}

Several limitations within the area make it challenging to develop guidance on food variety to assist with healthfully achieving energy balance. The first limitation is the previously described lack of consistent definition of variety. However, for consumer guidance, the definition of variety should consider aspects of variety that consumers can understand and quantify. This may mean that guidance using an ingredient-based approach may be challenging, as consumers may not have access to ingredients in mixed dishes that are consumed, or that the level of detail required in self-monitoring this approach may be too cumbersome. Thus, definitions of variety may need to take into account what consumers may be able to understand and use. It is not clear at this time what aspects of variety consumers understand and can quantify, as this has never been a component of previous investigations in the area.

Furthermore, detail is required about the degree of change in variety that is needed to impact energy intake to achieve energy balance. For example, guidance that indicates to increase and/or decrease variety is vague from a behavioral perspective and doesn't fit within guidelines for setting behavioral goals [54]. Ideally, guidance would include information related to the number of different items, however defined, to consume within an eating occasion, food group, and/or the overall diet. Moreover, as it is hypothesized that variety within different types of food, predominantly foods of differing energy densities, may moderate the relationship between food and energy intake and energy balance, guidance may need to include differing levels of 
variety for differing types of foods consumed. Finally, given the energy imbalance observed among US children and adults, all guidance on variety, similar to other dietary guidance, should include clear recommendations with respect to serving size and frequency of intake.

\section{Conclusions}

While it is clear that food variety influences energy intake, there are many gaps in the literature that need to be addressed to be able to develop guidance regarding food variety, food and energy intake, and energy balance (see Table 1). These gaps include how to define and measure food variety; understanding if type of food, particularly in regards to energy density, moderate the relationship between these variables; and practical guidance about food variety that consumers can understand and use to make change in their behavior than can influence food and energy intake and energy balance. 
Table 1: Evidence-based recommendations for dietary variety and future research needs

\begin{tabular}{|c|c|c|}
\hline $\begin{array}{l}\text { Dietary Variety } \\
\text { Goals/Guidance }\end{array}$ & $\begin{array}{l}\text { Current } \\
\text { Evidence Base }\end{array}$ & Future Research Needs \\
\hline $\begin{array}{l}\text { Limit variety } \\
\text { within HED } \\
\text { foods }\end{array}$ & $\begin{array}{l}\text { Preponderance } \\
\text { of evidence in } \\
\text { adults and } \\
\text { children } \\
\text { suggests that } \\
\text { greater variety } \\
\text { in HED foods is } \\
\text { positively } \\
\text { associated with } \\
\text { energy intake } \\
\text { and energy } \\
\text { imbalance } \\
\text { promoting } \\
\text { excess weight. }\end{array}$ & 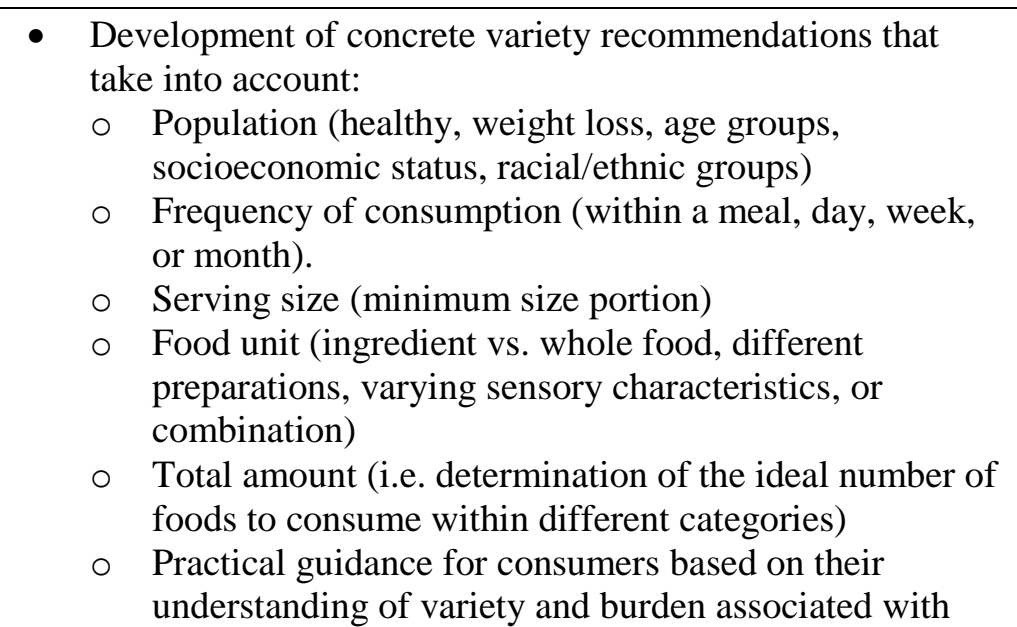 \\
\hline $\begin{array}{l}\text { Consume a } \\
\text { greater variety } \\
\text { of LED foods. }\end{array}$ & $\begin{array}{l}\text { Evidence in } \\
\text { adults and } \\
\text { children } \\
\text { suggests that } \\
\text { greater variety } \\
\text { in LED foods } \\
\text { (e.g. fruits and } \\
\text { vegetables) is } \\
\text { mixed in } \\
\text { regards to } \\
\text { energy intake } \\
\text { and non- } \\
\text { significantly or } \\
\text { inversely } \\
\text { associated with } \\
\text { excess weight }\end{array}$ & $\begin{array}{l}\text { - Development of a dietary assessment tool that precisely } \\
\text { examines variety over multiple days. } \\
\text { - Influence of } \\
\text { O Limiting HED variety on food cravings } \\
\text { Increasing LED variety on food substitution (i.e. } \\
\text { replacing HED foods with LED foods) } \\
\text { Concrete LED and HED variety prescriptions on energy } \\
\text { balance } \\
\text { - More longitudinal research } \\
\text { - Additional research examining moderators of variety }\end{array}$ \\
\hline
\end{tabular}


1

3

5

6

7

\section{References}

1. Raynor, H.A. and L.H. Epstein, Dietary variety, energy regulation, and obesity. Psychological Bulletin, 2001. 127: p. 325-341.

2. Kennedy, E., Dietary diversity, diet quality, and body weight regulation. Nutr Rev, 2004. 62(7 Pt 2): p. S78-81.

3. Epstein, L.H., et al., Habituation as a determinant of food intake. Psychological Reviews, 2009. 116: p. 384-407.

4. Beatty, W.W., Dietary variety stimulates appetite in females but not in males. Bulletin of the Psychonomic Society, 1982. 19: p. 212-214.

5. Bellisle, F. and J. Le Magnen, The analysis of human feeding patterns: The edogram. Appetite, 1980. 1: p. 141-150.

6. Bellisle, F. and J. Le Magnen, The structure of meals in humans: Eating and drinking patterns in lean and obese subjects. Physiology \& Behavior, 1981. 27: p. 649-658.

7. Bernstein, M.A., et al., Higher dietary variety is associated with better nutritional status in frail elderly people. J Am Diet Assoc, 2002. 102(Title): p. 1096-1104.

8. Berry, S.L., W.W. Beatty, and R.C. Klesges, Sensory and social influences on ice cream consumption by males and females in a laboratory setting. Appetite, 1985. 6: p. 41-45.

9. Epstein, L.H., et al., Food characteristics, long-term habituation and energy intake. Laboratory and field studies. Appetite, 2013. 60: p. 40-50.

10. Guerrieri, R., C. Nederkoorn, and A. Jansen, How impulsiveness and variety influence food intake in a sample of healthy women. Appetite, 2007. 48: p. 119-122. 
22 11. Guerrieri, R., C. Nederkoorn, and J. Jansen, The interaction between impulsivity and a varied food environment: Its influence on food intake and overweight. International Journal of Obesity, 2008. 32: p. 708-714.

12. Hetherington, M., B.J. Rolls, and V.J. Burley, The time course of sensory-specific satiety. Appetite, 1989. 12: p. 57-68.

13. Hollis, J.H. and C.J.K. Henry, Dietary variety and its effect on food intake of elderly adults. Journal of Human Nutrition and Dietetics, 2007. 20: p. 345-351.

14. $\quad •-$ Lock, C., et al., Contextual and environmental influences on reported dietary energy intake at evening eating occasions. Eating Behaviors, 2016. 21: p. 155-160. This is the first study to examine the influence of within-meal variety (served and consumed) on energy intake in a free-living setting. Results from this study suggest that the presence of food variety within a meal can enhance energy intake, providing support for the hypothesis that humans habituate to sensory input (even visually), and that this can influence food intake. This study also provides additional support that food type/energy density moderates the influence of variety on energy intake, with greater vegetable variety leading to reductions in energy intake within a meal.

15. Man-Mei Sea, M., et al., Associations between food variety and body fatness in Hong Kong Chinese adults. Journal of the American College of Nutrition, 2004. 23: p. 5.

16. Marshall, T.A., et al., Inadequate nutrient intakes are common and are associated with low diet variety in rural, community-dwelling elderly. Journal of Nutrition, 2001. 131: p. 2192-2196. 
43 17. Meengs, J.S., L.S. Roe, and B.J. Rolls, Vegetable variety: an effective strategy to increase vegetable intake in adults. Journal of the Academy of Nutrition and Dietetics, 2012. 112: p. 1211-1215.

46

18. Norton, G.N.M., A.S. Anderson, and M.M. Hetherington, Volume and variety: Relative effects on food intake. Physiology \& Behavior, 2006. 87: p. 714-722.

19. Oude Griep, L.M., et al., Variety in fruit and vegetable consumption and 10-year incidence of CHD and stroke. Public Health Nutrition, 2012. 15: p. 2280-2286.

20. Pliner, P., et al., Short-term intake of overweight individuals and normal weight dieters and non-dieters with and without choice amoung a variety of foods. Appetite, 1980. 1: p. 203-213.

21. Raynor, H.A., et al., Relationship between changes in food group variety, dietary intake, and weight during obesity treatment. International Journal of Obesity, 2004. 28: p. 813820.

22. Raynor, H.A., H.N. Niemeier, and R.R. Wing, Effect of limiting snack food variety on long-term sensory-specific satiety and monotony during obesity treatment. Eating Behaviors, 2006. 7: p. 1-14.

23. Raynor, H.A. and k.M. Osterholt, Greater variety of fruit served in a four-course snack increases fruit consumption. Appetite, 2012. 59: p. 662-667.

24. Raynor, H.A., et al., Limiting variety in non-nutrient-dense, energy-dense foods during a lifestyle intervention: A randomized controlled trial. American Journal of Clinical Nutrition, 2012.95: p. 1305-1314.

25. Raynor, H.A. and R.R. Wing, Effect of limiting snack food variety across days on hedonics and consumption. Appetite, 2006. 46: p. 168-176. 
66 26. Roberts, S.B., et al., Dietary variety predicts low body mass index and inadequate macronutrient and micronutrient intakes in community-dwelling older adults. The Journals of Gerontology. Series A, Biological Sciences and Medical Sciences, 2005. 60: p. 613-621.

70

27. Roe, L.S., et al., Serving a variety of vegetables and fruit as a snack increased intake in preschool children. American Journal of Clinical Nutrition, 2013. 98: p. 693-699.

28. Rolls, B.J., et al., Sensory specific satiety in man. Physiology \& Behavior, 1981. 27: p. 137-142.

29. Rolls, B.J., et al., Variety in a meal enhances food intake in man. Physiology \& Behavior, 1981. 26: p. 215-221.

30. Rolls, B.J., E.A. Rowe, and E.T. Rolls, How sensory properties of foods affect human feeding behavior. Physiology \& Behavior, 1982. 29: p. 409-417.

31. Rolls, B.J., v.D.P. M., and E.T. Rolls, Pleasantness changes and food intake in a varied four course meal. Appetite, 1984. 5: p. 337-348.

32. Royo-Bordonada, M.A., et al., Greater dietary variety is assodated with better biochemical nutritional status in Spanish children: The Four Provinces Study. Nutrition, Metabolism and Cardiovascular Diseases, 2003. 13: p. 357-364.

33. Spiegel, T.A. and E. Stellar, Effects of variety on food intakeof underweight, normalweight and overweight women. Appetite, 1990. 15: p. 47-61.

34. Torheim, L.E., et al., Nutrient adequacy and dietary diversity in rural Mali: Association and determinants. European Journal of Clinical Nutrition, 2004. 58: p. 594-604.

35. $\quad •$ Vadiveloo, M., et al., Use of a novel food diversity index to assess the role of dietary variety in body adiposity among adults. Journal of Nutrition, 2015. 145: p. 564-571. This 
study provides preliminary evidence from the National Health and Nutrition Examination Survey that the influence of variety on measures of body adiposity is moderated by factors like healthfulness/energy density and portion size.

36. $\quad \bullet$ Vadiveloo, M., et al., Increasing healthful dietary variety is associated with greater long-term reductions in adiposity among weight-loss participants in the POUNDS Lost trial. Journal of Nutrition, 2016. 146: p. 1552-1559. This study replicates evidence from the National Health and Nutrition Examination Survey in a longitudinal weight-loss population, and provides further support for the hypothesis that the influence of variety on measures of body adiposity is moderated by factors like healthfulness/energy density and portion size.

37. -Wijnhoven, H.A.H., B.S. van der Meij, and M. Visser, Variety within a cooked meal increases meal energy intake in older women with a poor appetite. Appetite, 2015. 95: p. 571-576. This study supports exisiting evidence that greater variety within a meal across a number of days can enhance energy intake, including among frail older women. This effect was observed among meals where energy density was held constant, with foods presented simultaneously rather than successively.

38. Woon Mok, L., The effect of variety and dietary restraint on food intake in lean young women: A preliminary study. The Journal of General Psychology, 2010. 137: p. 63-83.

39. Drewnowski, A., et al., The Dietary Variety Score: assessing diet quality in healthy young and older adults. J Am Diet Assoc, 1997.97(3): p. 266-71.

40. Remick, A.K., J. Polivy, and P. Pliner, Internal and external moderators of the effect of variety on food intake. Psychological Bulletin, 2009. 135(3): p. 434-451. 
111 41. Vadiveloo, M.K. and N. Parekh, Dietary Variety: An Overlooked Strategy for Obesity

112

113

114

115

116

117

118

119

120

121

122

123

124

125

126

127

128

129

130

131

132

133

134

and Chronic Disease Control. Am J Prev Med, 2015. 49(6): p. 974-9.

42. Epstein, L.H., et al., What constitutes food variety? Stimulus specificity of food. Appetite, 2010. 54(1): p. 23-9.

43. Rolls, B.J., The relationship between dietary energy density and energy intake. Physiology \& Behavior, 2009. 97: p. 609-615.

44. Bontrager Yoder, A.B. and D.A. Schoeller, Fruits and vegetables displace, but do not decrease, total energy in school lunches. Childhood Obesity, 2014. 10: p. 357-364.

45. Bond, D.S., et al., Salivary habituation to food stimuli in successful weight loss maintainers, obese and normal-weight adults. International Journal of Obesity, 2010. 34: p. 593-596.

46. Epstein, L.H., et al., Variety influences habituation of motivated behavior for food and energy intake in children. American Journal of Clinical Nutrition, 2009. 89: p. 746-754.

47. Epstein, L.H., et al., Long-term habituation to food in obese and nonobese women American Journal of Clincal Nutrition, 2011. 94(2): p. 371-376.

48. Raynor, H.A., Can limiting dietary variety assist with reducing energy intake and weight loss? Physiology \& Behavior, 2012. 106: p. 356-361.

49. Myers, M.E. and L.H. Epstein, Habituation of responding for food in humans. Appetite, 2002. 38: p. 224-234.

50. Temple, J.L., et al., Dietary variety impairs habituation in children. Health Psychology, 2008. 27: p. S10-S19.

51. Epstein, L.H., et al., Slow rates of habituation predict greater zBMI gains over 12 months in lean children. Eating behaviors, 2011. 12(3): p. 214-218. 
135 52. Temple, J.L., et al., Habituation and within-session changes in motivated responding for 136 food in children. Appetite, 2008. 50: p. 390-396.

137 53. Murphy, S.P., et al., Simple measures of dietary variety are associated with improved 138 dietary quality. J Am Diet Assoc, 2006. 106(3): p. 425-9.

139 54. Spahn, J.M., et al., State of the evidence regarding behavior change theories and 140 strategies in nutrition counseling to facilitate health and food behavior change. Journal of 141 the American Dietetic Association, 2010. 110: p. 879-891. 